\title{
Evaluation of Instrumental Errors Built in Circular Dichroism Spectrometers
}

\author{
ETTORE CASTIGLIONI, ${ }^{1,2 *}$ PAOLO ALBERTINI, ${ }^{3}$ AND SERGIO ABBATE ${ }^{2,4}$ \\ ${ }^{1} J A S C O$ Corporation, Ishikawa-cho, Hachioji-Shi, Tokyo 192-8537, Japan \\ ${ }^{2}$ Dipartimento di Scienze Biomediche e Biotecnologie, Università di Brescia, 25123 Brescia, Italy \\ ${ }^{3}$ JASCO Europe, 23894 Cremella, Italy \\ ${ }^{4}$ CNISM, Consorzio Nazionale Interuniversitario Scienze Fisiche della Materia, \\ 00146 Roma, Italy
}

Contribution to the CD ISBC 2009 Special Issue

\begin{abstract}
Because of the increased use of circular dichroism (CD) spectroscopy as a routine technique by nonspecialists to determine the conformational/configurational properties of biomolecules, we have decided to present here some criteria to accurately check the ordinate scale calibration of a CD spectrometer particularly in the critical lowwavelength UV region, to understand, and correct, where possible, the potential limitations coming from the hardware. We also analyze some wavelength calibration methods, and some standards for the CD-scale calibration, and we discuss the critical characteristics of current instrumentation affecting measurements. The example of the bovine catalase CD spectrum is considered. Chirality 22:E142-E148, 2010. @ @ 2010 Wiley-Liss, Inc.
\end{abstract}

KEY WORDS: circular dichroism; calibration procedures; instrumental limitations; wavelength accuracy; bovine catalase

\section{INTRODUCTION}

About $80 \%$ of the circular dichroism (CD) spectrometers installed worldwide are used to analyze biomolecules. The technique has spread out remarkably and is mainly utilized as a complementary analytical tool mostly by nonspecialists. To confirm the accuracy of the obtained results, one has to first better understand the potential limitations of instruments and operations. This is particularly true today because measurements are also carried out with new approaches, based, e.g., on the use of synchrotron radiation beamlines, which allow the wavelength range to be extended into the critical far UV region. However, CD calibration has been and still is a matter of dispute. An early study on a large number of $\mathrm{CD}$ spectrometers evidenced, many years ago, ${ }^{1}$ an unexpected scattering of results. Despite considerable advancement in the instrumentation, a similar recent survey of the UV and far UV range by the UK National Physical Laboratory (NPL) ${ }^{2}$ reported similar inconsistencies.

The possible error sources in CD measurements fall in three main categories:

1. Sample preparation and improper sampling procedures,

2. Incorrect measuring parameters, and

3. Instrumental errors.

Because the first two topics have been widely discussed in several review articles, ${ }^{3-5}$ we will concentrate on the third theme; indeed, we think that a systematic approach to the instrumental source of errors is still missing (All measurements and tests for this work have been carried out using recent JASCO spectrometers, but our approach is for sure applicable also to older JASCO units or to spectrometers by other manufacturers. The only requisites for these machines are the possibility to collect and record the DC signal and an installed facility to switch the photomultiplier tube into manual, i.e., excluding dynode feedback. If these options are not available, we strongly suggest that a service engineer implements them.) In dealing with this theme, three main problems need to be tackled: (i) checking wavelength accuracy; (ii) finding good calibration standards; and (iii) identifying intrinsic instrumental limitations.

\section{CHECKING WAVELENGTH ACCURACY}

A CD spectrometer can be considered as a normal UVVis spectrophotometer and consequently very similar approaches to those employed in UV-Vis spectroscopy can be used to verify wavelength scale calibration with readily available commercial standards. ${ }^{6,7}$ The latter however typically are not fit for the far-UV region, for which an appropriate calibration standard is the $\mathrm{NH}_{3}$ vapor spectrum, ${ }^{8}$ which is easy to obtain in a gas tight cell. A simple alternative is to use the Schumann-Runge $\mathrm{O}_{2}$ absorption bands ${ }^{9}$; in this case, it is enough to operate the instrument with incomplete $\mathrm{N}_{2}$ purging (or purging with low-purity nitrogen as in our case) and to collect data using a narrow slit band-width (SBW) value such as $\leq 0.1 \mathrm{~nm}$, to obtain a spectrum with

\footnotetext{
*Correspondence to: Ettore Castiglioni, JASCO Corporation, Ishikawacho, Hachioji-Shi, Tokyo 192-8537, Japan.

E-mail: ettore.castiglioni@jasco-europe.com

Received for publication 5 November 2009; Accepted 1 March 2010

DOI: $10.1002 /$ chir.20860

Published online 28 April 2010 in Wiley Online Library

(wileyonlinelibrary.com).
} 


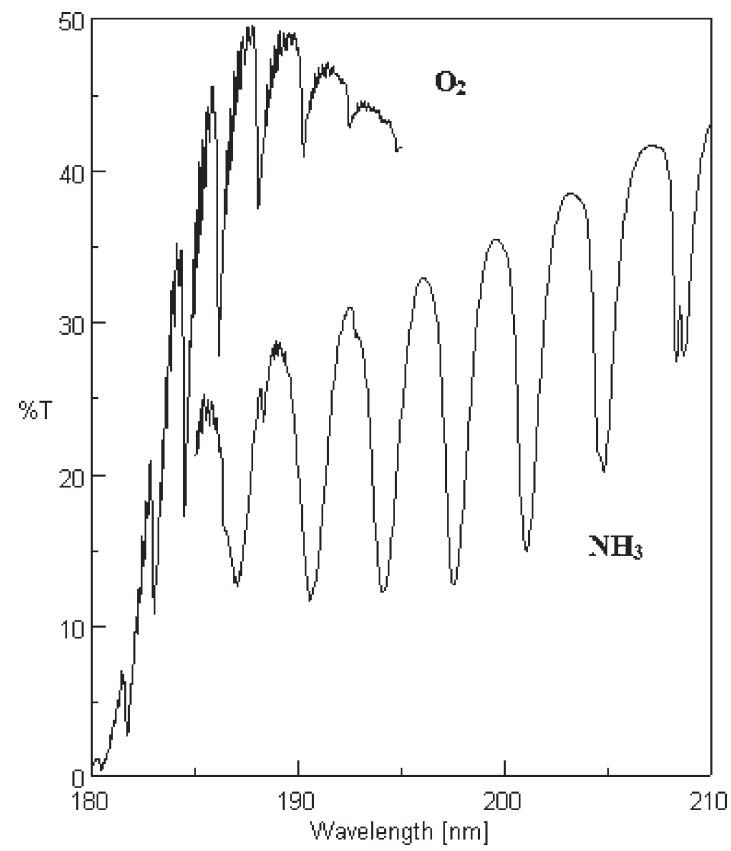

Fig. 1. Single beam high resolution $\% T$ spectra of $\mathrm{O}_{2}$ gas $(\mathrm{SBW}<0.1$ $\mathrm{nm})$ and of $\mathrm{NH}_{3}$ vapor (SBW $\left.\leq 0.2 \mathrm{~nm}\right)$, arbitrarily scaled for better readability. The band-center wavelengths of the observed bands for $\mathrm{O}_{2}$ are 181.7, 183.0, 184.5, 186.4, 188.1, 190.2, $192.4(\mathrm{~nm})$ and for $\mathrm{NH}_{3}, 187.2,190.6,194.0$, 197.5, 201.1, 204.8, $208.6(\mathrm{~nm})$. Because the $\mathrm{O}_{2}$ bands are superposed on an absorbing continuum that is strongly increasing with decreasing wavelength, a slight shift on the observed readings may take place.

sharp bands and well-defined wavelengths. Figure 1 shows the two spectra and lists the most useful wavelength values of these two samples; suitable bandpasses are indicated, because exact band positioning, particularly with gas phase samples, is related to the used SBW.

A recent book devoted to $\mathrm{CD}$ includes an article by Sutherland, ${ }^{10}$ which shows wavelength calibration data of a $\mathrm{CD}$ synchrotron beamline using $\mathrm{N}_{2}$ (for the range below $150 \mathrm{~nm}$ ) and $\mathrm{O}_{2}$ vapor absorption lines, as well as holmium oxide in perchloric acid solution with bands above $240 \mathrm{~nm}$. Another chapter ${ }^{11}$ of the same book reports also benzene vapor as wavelength calibration standard in the 265-240 $\mathrm{nm}$ region: this is a widely used standard in the UV region, ${ }^{12}$ but data reported in Ref. 11 are somehow misprinted. Wavelength accuracy affects the quality not only of CD spectra, but also of any other spectra, and, due to this, we are sure that the interested reader may find a large amount of literature on this topic and we will stop here on this part. For similar reasons, we will not describe procedures for hardware recalibration, because the matter is dealt with in the instruments service manuals and recalibration should be performed by an expert service engineer.

\section{SCALE STANDARDS}

While the ordinate scale calibration of a UV-Vis spectrophotometer is usually checked at a single wavelength, in the $\mathrm{CD}$ case this is not sufficient due to the chromatic characteristic of the photoelastic modulator (PEM) employed in all $\mathrm{CD}$ spectrometers and due to the nature of the $\mathrm{CD}$ signal, which depends both on the chirality and the absorption of the sample. In contrast to the optical rotary dispersion technique, which can be measured with optically null spectropolarimeters, CD spectrometers cannot provide absolute measurements. Different reference materials have been proposed to overcome this limitation: epiandrosterone in dioxane, with a CD band at $304 \mathrm{~nm},{ }^{13} \mathrm{D}$-10-camphorsulfonic acid (CSA) ${ }^{14,15}$ or ammonium-d-camphorsulfonate (ACS) samples ${ }^{16}$ in water. The latter provides not only the conventional check at $290.5 \mathrm{~nm}$, but, even more important, allows one to measure the proper intensity ratio (about 2 ) of the negative band at $192 \mathrm{~nm}$ with respect to the positive one at $290.5 \mathrm{~nm}$. This further allows one to confirm (or otherwise) the linearity of the PEM program (see Fig. 2). This is the linearity calibration method suggested by European Pharmacopoeia (EP), ${ }^{17}$ which indicates a very broad acceptance range for the value, from 1.72 to 2.27 (while the commonly accepted ratio today ranges from 2 to 2.1). For the $\mathrm{CD}$ scale, the EP recommends epiandrosterone in dioxane (reported as isoandrosterone $\mathrm{R}$ in dioxane $\mathrm{R}$ in their document) at $304 \mathrm{~nm}$. A more elaborate calibration of the PEMs has been published, ${ }^{18}$ but the latter requires use of an analyzer in the light beam and employing an oscilloscope and specific skill.

An important advantage of the ACS/CSA approach consists in the traceability to their absolute optical rotation measurements via the Kramers-Kronig relation..$^{19}$ Certified ACS standards are commercially available from JASCO, with enantiomeric purity checked by a validated optically null polarimeter.

Many other chemical calibrants have been proposed and are often used: D-pantolactone in water with a CD

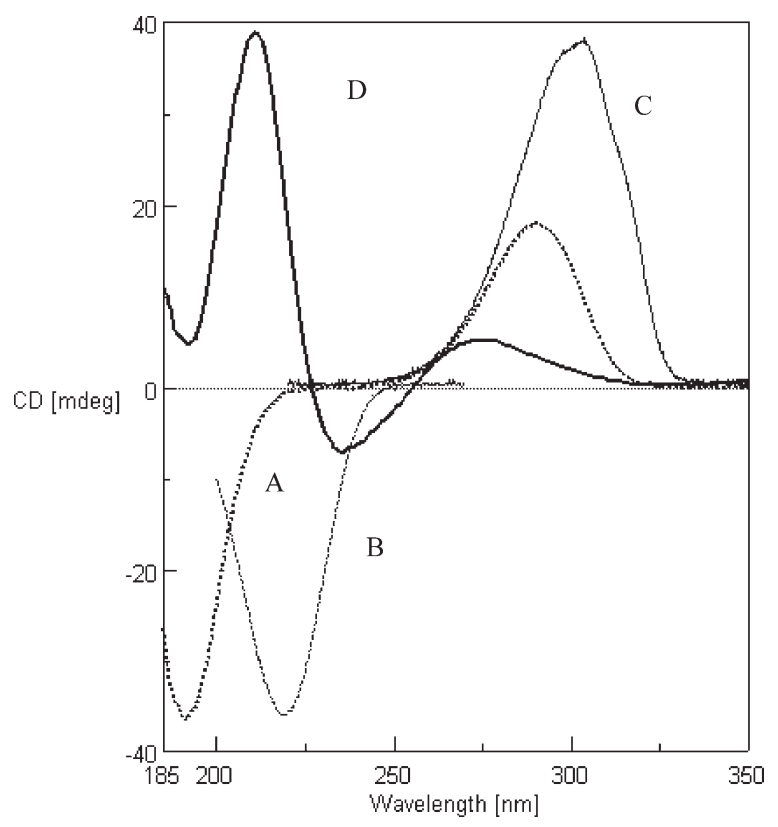

Fig. 2. Superimposed CD spectra for a few $C D$ scale standards for the UV range. (A) Ammonium d-10 camphorsulfonate $(60 \mathrm{mg} / 100 \mathrm{ml}$ in water, 1-mm cell); (B) D-(-)-Pantolactone (3 mg/100 $\mathrm{ml}$ in water, 10-mm cell); (C) Epiandrosterone (50 $\mathrm{mg} / 100 \mathrm{ml}$ in dioxane, 10-mm cell); and (D) $\mathrm{Na}[\mathrm{Co}(\mathrm{EDDS})]$ (0.056 $\mathrm{mM}$ in water, $10-\mathrm{mm}$ cell). 
band at $220 \mathrm{~nm},{ }^{20} \Delta-(-)-\mathrm{Co}(\mathrm{en})_{3} \mathrm{I}_{3} \mathrm{H}_{2} \mathrm{O}$ in water with a main band in the visible at $490 \mathrm{~nm},{ }^{21,22}$ nickel (II) tartrate for the Vis-NIR range. ${ }^{23}$ Other suggested standards include the adamantane crystal proposed by Snatzke (Günther Snatzke personal communication to EC during 1985 CD conference in Sofia), which had batch to batch reproducibility problems, and the optical device patented by Steinberg ${ }^{24}$; neither of these approaches found widespread practical applications. The recent article by Tanaka $^{25}$ presents the design of a "polarimeter" as a calibration tool for AC-modulated polarizing undulators, which may be extended to conventional PEM systems, but the use of this "device" looks complex. The conventional CD scale calibration approach of IR CD spectrometers, ${ }^{26}$ based on the use of a second polarizer and a birefringent plate, in principle could be employed in the UV-visible regions of the spectrum, but is suitable only for very strong equivalent $\mathrm{CD}$ signals and is difficult to implement in the far UV region. The goal of absolute calibration was pursued by Schippers and Dekkers ${ }^{27}$ using an ad hoc assembled CD apparatus, whereas Nordén and $\mathrm{Seth}^{28}$ proposed an absolute method of calibrating the $\mathrm{CD}$ scale based on the linear dichroism (LD) response calibrated with tilted quartz plates. We wish to point out that long time ago Holzwarth and Doty ${ }^{29-31}$ had suggested that the absolute CD measurements obtained using a thin quartz retardation plate might be more accurate than those obtained with an electro-optic modulator. The approach of Holzwarth and Doty however suffered from the fact that $\mathrm{CD}$ values were obtained for a finite number of wavelengths and was rarely applied afterward. ${ }^{32}$ More recently, the Wallace group has investigated the calibration problem of both conventional and synchrotron radiation CD spectrometers. ${ }^{33,34}$ Two new standards have been recently proposed: a chlorine dimer with two sharp exciton doublets in the UV-Vis ${ }^{35}$ range and a compound ( $\mathrm{Na}[\mathrm{Co}(\mathrm{EDDS})]$. $\left.\mathrm{H}_{2} \mathrm{O}\right),{ }^{36}$ which has several bands in the wavelength range of interest: long-term stability is excellent and both enantiomeric forms are available. The latter compound will shortly be available commercially to any user in a calibration kit with sealed cells for the two enantiomers and the solvent. The intrinsic limitation of this approach is that one cannot correct the related baseline, because the chiral samples and the blank (solvent) are contained in different cells, while good operating practice consists in the use of the same cell for sample and solvent. Needless to say, some of the above calibrants or calibration procedures for the $\mathrm{CD}$ scale also provide an approximate calibration for the wavelength scale.

The authors of Ref. 11 have proposed creating a calibration curve to correct spectral intensity of unknown samples via software, based on data obtained by measuring the CD scale errors at different wavelengths, e.g., by putting together CD data that are from several calibrants in the wavelength range of interest (as done, e.g., in Fig. 2). This approach looks attractive, but it does not take into account other sources of error as we will outline below and may therefore in some cases be no better than the original situation.

\section{INSTRUMENTAL LIMITATIONS AFFECTING CD SCALE, WHICH CANNOT BE AMENDED BY CALIBRATION}

Before going into details, it is necessary to recall the measuring principle employed in current $\mathrm{CD}$ instrumentation. All modern CD spectrometers are basically single beam spectrophotometers in which the linearly polarized radiation from a monochromator is converted into periodically circularly polarized radiation by a PEM operating at about $50 \mathrm{KHz}$ (in the UV-visible region). $\mathrm{CD}$ is typically measured as the $\mathrm{AC} / \mathrm{DC}$ ratio, where $\mathrm{AC}$ is the lock-in amplifier output synchronically linked to the PEM oscillation (being non-zero only if the sample is CD active), and DC is a continuous signal proportional to instrumental light-flux and to sample plus buffer transmittance. Practically in all commercially available units, the DC level is kept constant by the photomultiplier (PM) tube dynode feedback and thus current instruments allow one to measure only the phaselinked intensity of the AC component. To give a specific example, in the JASCO J-815 case, the DC level is kept automatically at $1 \mathrm{~V}$ (see Fig. 3). With the CD scale calibrations described above, we are able to easily check proper intensity for the $\mathrm{AC}$ component, but what about the DC one? Because of the $\mathrm{AC} / \mathrm{DC}$ relationship, overestimate of the $\mathrm{DC}$ level results in an artificial decrease of the $\mathrm{CD}$ band intensity, while underestimate of the DC level results in an artificial increase of the CD band intensity. Let us now assume that we are dealing with a properly prepared sample in a perfectly homogeneous solution, with no scattering or absorption flattening side effects, which may distort the sample apparent transmittance and consequently the DC level. ${ }^{37}$ What are

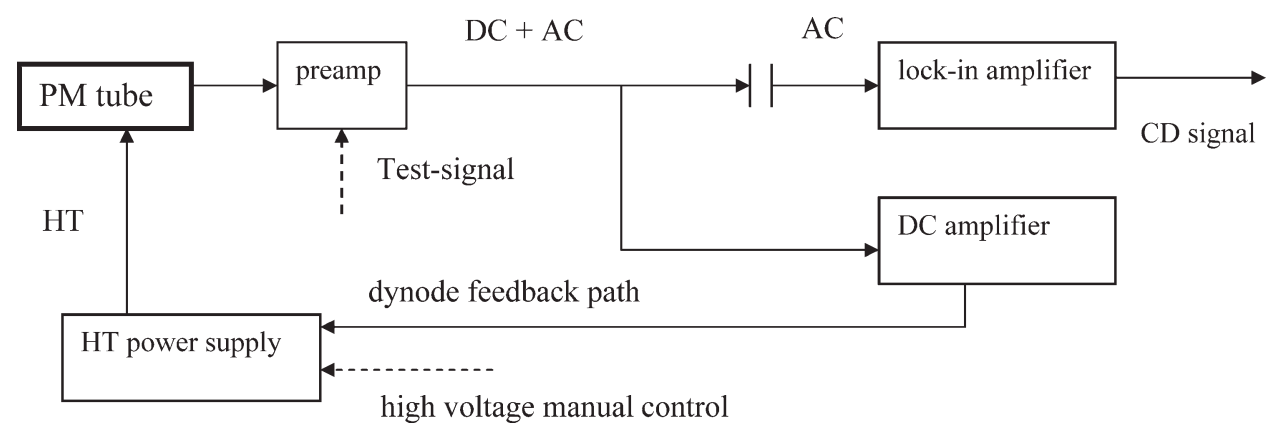

Fig. 3. Block diagram of AC and DC signal treatment by current JASCO CD spectrometers. This scheme is not exclusive of JASCO manufacturer. 
TABLE 1. Evaluation of ambient stray light and dark current influence on the DC signal, as measured from three JASCO instruments (see text)

\begin{tabular}{|c|c|c|c|c|c|c|}
\hline \multirow[b]{2}{*}{$\begin{array}{l}\text { PM tube } \\
\text { voltage } \\
\text { (V) }\end{array}$} & \multicolumn{2}{|c|}{ J-815(1) } & \multicolumn{2}{|c|}{$\mathrm{J}-815(2)$} & \multicolumn{2}{|c|}{ J-815SE } \\
\hline & $\begin{array}{l}\text { Room } \\
\text { light on } \\
(\mathrm{mV})\end{array}$ & $\begin{array}{l}\text { Room } \\
\text { light off } \\
(\mathrm{mV})\end{array}$ & $\begin{array}{l}\text { Room } \\
\text { light on } \\
(\mathrm{mV})\end{array}$ & $\begin{array}{l}\text { Room } \\
\text { light off } \\
(\mathrm{mV})\end{array}$ & $\begin{array}{l}\text { Room } \\
\text { light on } \\
(\mathrm{mV})\end{array}$ & $\begin{array}{l}\text { Room } \\
\text { light off } \\
(\mathrm{mV})\end{array}$ \\
\hline 0 & 0 & 0 & 0 & 0 & 0 & 0 \\
\hline 200 & 2 & 2 & 2 & 2 & 1 & 1 \\
\hline 300 & 3 & 2 & 3 & 2 & 1.5 & 1.5 \\
\hline 400 & 5 & 4 & 3 & 3 & 2 & 2 \\
\hline 500 & 6 & 5 & 5 & 4 & 3 & 3 \\
\hline 600 & 10 & 7 & 8 & 6 & 4 & 4 \\
\hline 700 & 20 & 9 & 23 & 11 & 5 & 4.5 \\
\hline 800 & 43 & 10 & 52 & 24 & 6 & 5 \\
\hline 900 & 80 & 14 & 96 & 46 & 11 & 8 \\
\hline
\end{tabular}

The DC signal in normal operation is kept at $1000 \mathrm{mV}$ by dynode feedback.

the factors that may adversely influence the DC measurement and consequently the high voltage (HT) applied to the PM? For simplicity let us assume use of a spectrometer able to measure $\mathrm{AC}$ and $\mathrm{DC}$ separately, assigning a fixed voltage to the $\mathrm{PM}$, with the correct $\mathrm{CD}$ evaluated as the $\mathrm{AC} / \mathrm{DC}$ ratio afterwards (this is different from how instruments currently work as in Fig. 3, which is standard also for JASCO spectrometers; however, this alternative mode may be chosen in most recent JASCO instruments). There are four possible reasons for incorrect $\mathrm{DC}$ evaluation:

1. Wrong offset of the DC preamplifier;

2. PM tube dark current;

3. Ambient stray-light feeding the detector; and

4. Spectrometer stray light.

As mentioned above, modern CD spectrometers measure DC spectra as single-beam spectrometers do, so reasons 1-3 may give erroneous offset DC signals even when the source-lamp is off. While this drawback was pointed out several years ago and corrected by modulating the light with a mechanical chopper, ${ }^{38}$ manufacturers decided to keep the DC approach because high sensitivity, which is proportional to the square root of the light flux, is needed in a CD measurement. Let us now analyze points 1 through 4 .

\section{Wrong Offset of DC Preamplifier}

It is typically very low and it is easy to compensate by a simple trimmer operation, relevant information is available in the service manuals.

\section{Dark Current of PM Tube}

It is a signal related to the high voltage applied on the PM tube dynodes (HT). Any PM tube generates dark current and the dark current level increases with HT. The latter voltage normally is higher at low wavelengths.

\section{Ambient Stray-Light Feeding the Detector}

Sample compartments are properly light sealed; yet, even without user interference, at very high-applied voltage, even minuscule amounts of ambient light can be detected. This problem was observed and taken into account, in the field of chiral spectroscopy, a long time ago. ${ }^{39}$

We have tested the DC signal, with the light source switched off in a normal laboratory environment with either just daytime ambient light or switching on also the room light (a fluorescent lamp). Three JASCO CD spectrometers have been tested: two standard J-815 and a J-815SE, the latter being a stripped down variant of J-815. All three units use the Hamamatsu R376 PM tube, but the PM tube mounted on J-815SE is nearly 30 years old. The new mounting on the J-815SE apparatus was however optimized to use very small solid angle light collection. Table 1 reports the data obtained and one may see that for the J-815 machine, where the DC level in normal dynode feedback operation is $1 \mathrm{~V}$, errors may exceed $1 \%$, when the dynode voltage is above $600 \mathrm{~V}$.

\section{Spectrometer Stray Light}

To check the amount of stray-light from inside the spectrometer we followed the American Society for Testing Materials $^{40}$ (ASTM) prescription and took a spectrum of $12 \mathrm{~g} / 1$ $\mathrm{KCl}$ aqueous solution in a $1-\mathrm{cm}$ pathlength cell. This standard is very valuable because it allows us to measure the straylight in the low-wavelength UV region, corresponding to the $\pi \rightarrow \pi^{*}$ transition of polypeptides. We carried out the measurement on our JASCO J-815SE equipped with a Xe source which had already been used for about $500 \mathrm{~h}$; we worked with $5 \mathrm{l} / \mathrm{min}$ flux of $\mathrm{N}_{2}$ purging gas and $1 \mathrm{~nm}$ bandpass. A single beam $\mathrm{DC}$ spectrum of air $\left(\mathrm{DC}_{\text {Air }}\right)$ was collected at fixed $250 \mathrm{~V}$ PM voltage (HT) over the whole $225-182 \mathrm{~nm}$ range. Then, the $\mathrm{DC}$ spectrum of the $\mathrm{KCl}$ solution was measured in three segments: from 225 to $201 \mathrm{~nm}$ at $250 \mathrm{~V}$ HT $\left(\mathrm{DC}_{\mathrm{KCl250}}\right)$, from 201 to $199 \mathrm{~nm}$ the HT was increased to 500 $\mathrm{V}$ (DC gain about $200 \times)$ to expand the signal $\left(\mathrm{DC}_{\mathrm{KC} 1500}\right)$; from 199 down to $182 \mathrm{~nm}$ the HT was further increased to $900 \mathrm{~V}$ (DC gain raised another $100 \times)\left(\mathrm{DC}_{\mathrm{KCl} 1900}\right)$. Last, the DC signal with light source off and HT at $900 \mathrm{~V}$ was collected from 199 to $182 \mathrm{~nm}\left(\mathrm{DC}_{\text {Dark900}}\right)$. Figure 4 (left) shows the
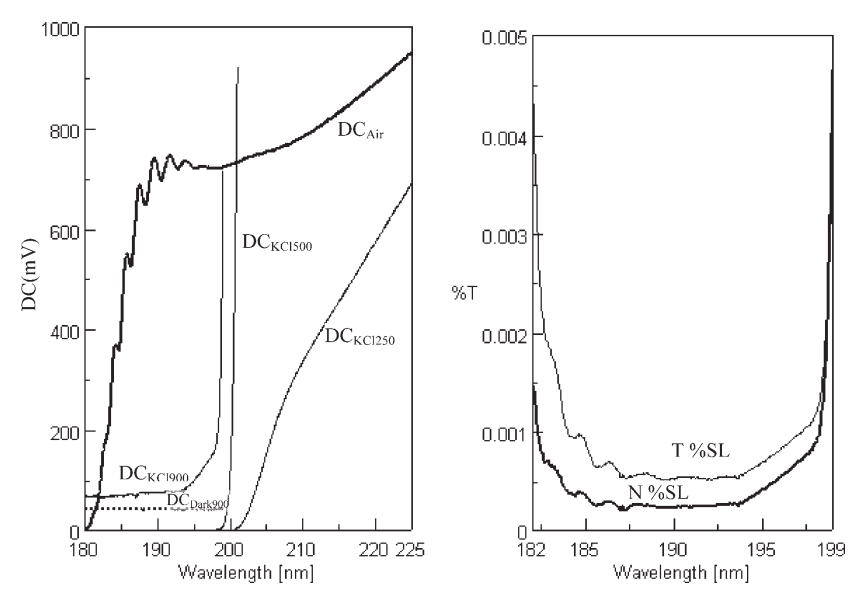

Fig. 4. Left: Measurement of stray-light with $\mathrm{KCl}$ aqueous solution according to ASTM method, see text for details. Thick line: air blank at $250 \mathrm{~V}$; thin lines: $\mathrm{KCl}$ solution at 250, 500, and $900 \mathrm{~V}$, dotted line PM dark current at $900 \mathrm{~V}$. Right: computed total stray-light (T\%SL), thin line, and net spectrometer stray-light ( $N \% \mathrm{SL})$, thick line (see text). 
five DC spectra collected in this way, the waving in the $\mathrm{DC}_{\mathrm{Air}}$ spectrum being due to oxygen contamination (unresolved Schumann-Runge bands) in the nitrogen supply.

The amount of stray-light can then be evaluated by taking into account the total gain change $(200 \times 100=$ 20,000 ) by defining the total percentage amount of straylight $(T \% \mathrm{SL})$ and the net percentage stray-light level originating from the spectrometer $(N \% \mathrm{SL})$ respectively as:

$$
\begin{gathered}
T \% \mathrm{SL}=100\left[\mathrm{DC}_{\mathrm{KC} 1900} /\left(20,000 \times \mathrm{DC}_{\text {Air }}\right)\right] \\
N \% \mathrm{SL}=100\left[\left(\mathrm{DC}_{\mathrm{KC} 1900}-\mathrm{DC}_{\text {Dark900 }}\right) /\left(20,000 \times \mathrm{DC}_{\text {Air }}\right)\right]
\end{gathered}
$$

Figure 4 (right) shows the result of the latter operations: $N \% \mathrm{SL}$ of the spectrometer is at most $0.0002 \%$ at $190 \mathrm{~nm}$ and T\%SL reaches $0.0005 \%$ at most (in the latter quantity we consider also ambient stray-light and PM tube dark current). We wish to point out that the stray-light in the far-UV region is not a constant over the entire instrument lifetime: the stray-light level will significantly increase when the source ages, which typically means a decrease of far-UV emitted photons, and with the ageing of the source mirrors surfaces, because this reduces reflectivity mainly for the $\mathrm{UV}$ radiation. High quality $\mathrm{N}_{2}$-purging is also essential for
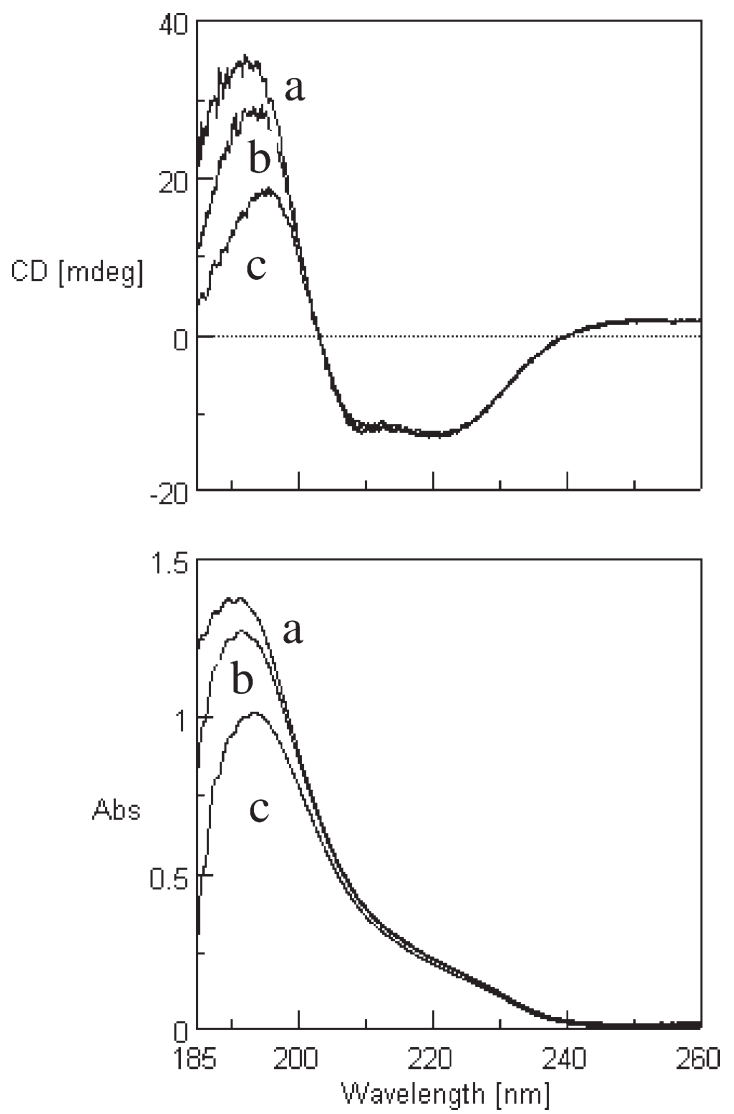

Fig. 5. CD (top) and absorption (bottom) spectra of $0.2 \mathrm{mg} / \mathrm{ml}$ bovine liver catalase solution ( $\mathrm{pH} 7.5$ ) in 1-mm cell, with the spectrometer operating in normal conditions (a), and admitting $0.5 \%$ (b) or $2 \%$ (c) amount of ambient light. CD baselines not subtracted.

Chirality DOI 10.1002/chir


Fig. 6. Superimposed percent transmittance and CD error spectra $(\Delta \% T$ and $\Delta \mathrm{CD}$, respectively), bold and thin lines admitting $2 \%$ (left) or $0.5 \%$ (right) amount of ambient light, respectively. Ordinate scale is in dimensionless units, being associated with the ratios $\% T / \% T$ and $\mathrm{CD} / \mathrm{CD}$ data, as of the definition (see text).

reducing stray-light: below $185 \mathrm{~nm}$ this is quite effective in the instruments we used. While one expects better performances from a new lamp and better purging, yet, even in a perfect brand-new instrument, stray-light is present at the wavelength limits, particularly in the far-UV, where Xe source emission is relatively weak.

To further evaluate the effects of stray-light and other DC errors discussed above, we have decided to perform a test on a typical biologically interesting sample, that is to say we took the $\mathrm{CD}$ spectrum of a $0.2 \mathrm{mg} / \mathrm{ml}$ solution of bovine liver catalase (PBS buffer at $\mathrm{pH} 7.5$ ) in a 1 -mm cell (single scan with $1 \mathrm{~nm}$ SBW, $20 \mathrm{~nm} / \mathrm{min}$ scanning speed and $1 \mathrm{sec}$ integration time). Distortions in the $\mathrm{CD}$ and in the absorption spectra were artificially induced by admitting two different amounts of ambient stray-light into the sample compartment. The admitted light levels were about $0.5 \%$ and $2 \%$ of the measurement light at $240 \mathrm{~nm}$, as determined by the DC signal at constant HT $=250 \mathrm{~V}$ applied to the PM.

We may notice in Figure 5 how both the $\mathrm{CD}$ and the absorption spectra are progressively distorted in intensity and wavelength in the low-wavelength UV band: the measured spectra are labeled (a) when taken in normal conditions $\left[\mathrm{CD}_{\text {Norm }}(\lambda)\right.$ and $\left.\mathrm{Abs}_{\text {Norm }}(\lambda)\right]$, (b) when taken with $0.5 \%$ perturbation from ambient stray-light $\left[\mathrm{CD}_{0.5 \%}(\lambda)\right.$ and $\left.\operatorname{Abs}_{0.5 \%}(\lambda)\right]$, and (c) when taken with $2 \%$ perturbation from ambient stray-light $\left[\mathrm{CD}_{2 \%}(\lambda)\right.$ and $\left.\mathrm{Abs}_{2 \%}(\lambda)\right]$.

Extracting the corresponding $\% T$ spectra from the absorbance data $\left[\% T_{\text {Norm }}(\lambda), \% T_{0.5 \%}(\lambda), \% T_{2 \%}(\lambda)\right]$ and calculating the ratios $\% T_{\text {Norm }}(\lambda) / \% T_{0.5 \%}(\lambda)$ and $\% T_{\text {Norm }}(\lambda) /$ $\% T_{2 \%}(\lambda)$, we can plot the error $\Delta T$ spectra in the two conditions. In the same way, we calculate the error spectra of the $\mathrm{CD}$ data $\left[\mathrm{CD}_{\text {Norm }}(\lambda) / \mathrm{CD}_{0.5 \%}(\lambda)\right.$ and $\operatorname{CD}_{\text {Norm }}(\lambda) /$ $\left.\mathrm{CD}_{2 \%}(\lambda)\right]$ and denote them $\Delta \mathrm{CD}$. Figure 6 shows the nearly perfect overlay of the $\mathrm{CD}$ and $\% T$ error spectra under both conditions and this proves that the major distortions of $\mathrm{CD}$ spectra originate directly from the artifacts present in the absorption ones. 
At this point, we can provide three basic suggestions:

- If one notices that absorption spectra are distorted, it is possible to correct the $\mathrm{CD}$ data by either assuming that the exact absorption spectrum is known, or by remeasuring it on a suitable UV-Vis spectrophotometer (The absorbance spectra reported in this work were collected by taking advantage of the facility present in many modern spectrometers, which allows us to obtain absorbance spectra from the PM tube high voltage (HT) in the dynode feedback mode by using the close to linear relation A versus HT stored in the spectrometer memory. An alternative way to collect absorbance spectra calls for the use of the DC scale at constant HT: DC spectra for the sample solution and for the blank (e.g., the solvent) may be scanned in this way, provided the HT is kept low enough not to saturate the DC signal. The $\% T$ spectra of the sample is then obtained by dividing the sample DC spectra by the blank DC spectra.) and using this as follows. Let us call such an absorption spectrum (in transmittance scale) $\% T_{\text {True }}(\lambda)$. From the measured $\mathrm{CD}$ and transmittance spectra $\operatorname{CD}_{\text {Meas }}(\lambda)$ and $\% T_{\text {Meas }}(\lambda)$, one can obtain the correct and thus true $C D$ spectrum, $\operatorname{CD}_{\text {True }}(\lambda)$. One has indeed:

$$
\mathrm{CD}_{\text {True }}(\lambda) / \mathrm{CD}_{\text {Meas }}(\lambda)=\% T_{\text {True }}(\lambda) / \% T_{\text {Meas }}(\lambda)
$$

and thus:

$$
\mathrm{CD}_{\text {True }}(\lambda)=\mathrm{CD}_{\text {Meas }}(\lambda)\left[\% T_{\text {True }}(\lambda) / \% T_{\text {Meas }}(\lambda)\right]
$$

- When publishing CD spectra, one should always provide also the absorption spectrum collected with the same machine at the same time (following the specific procedures of the instrument used). This used to be common practice several years ago, but nowadays is scarcely followed.

- One needs to verify spectral consistency by working at different sample concentrations and/or using shorter pathlength cells. Sample dilution is indeed the most effective and simplest way for a first check whether instrumental limitations are present.

The last source of instrumental error is due to possible saturation of the AC amplifier, which happens when, operating in the dynode feedback mode, the high voltage of the PM tube becomes very high. To practically test the working limits, we operated our J-815SE in the test signal mode, i.e., by feeding a synthetic AC signal at preamplifier level of about $18 \mathrm{mdeg}$. This facility is built into this spectrometer and is designed to check, during initial diagnostics, the proper operation of the whole photometric electronics when no voltage is applied to the PM tube. In our test, we fed a variable HT to the PM to see the effect of PM noise on the synthetic signal applied to the preamplifier. The results (Fig. 7) were very encouraging and instructive: even increasing the voltage up to $1000 \mathrm{~V}$, we obtained no loss of signal intensity, notwithstanding a substantial growth of the noise level in the CD signal. This proves that a very high voltage on PM tube is not the

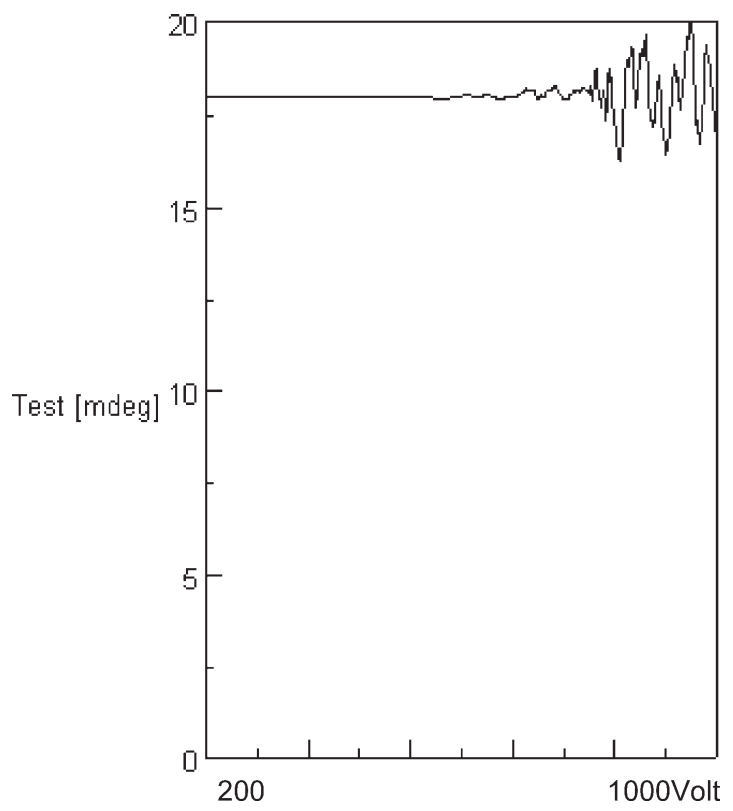

Fig. 7. Saturation test of the AC signal, performed by increasing the photomultiplier tube high voltage, see text for details.

cause of AC-signal preamp saturation, at least for properly designed photometric electronics.

\section{CONCLUSIONS}

Although periodic CD scale calibration checks using reference CD standards is a good practice, it may not be sufficient. CD spectra distortions originate, in many if not in most cases, from lack of accuracy in the measurement of the DC component. This is particularly true in the lowwavelength UV region, where spectrometers operate close to or above their limits.

Testing or calibrating by use of CD standards solutions is important, but it fails to give reliable compensation factors for possible software corrections, when the distortions are mainly related to:

- Actual sample (plus buffer) absorption levels,

- Ambient and spectrometer stray-light,

- Dark current of the PM tube,

- Proper conditions of the optical system (mirrors reflectivity, lamp emission) particularly in the far-UV range, and

- Suitable purging with water-free nitrogen gas.

Some of these imperfections increase with lack of maintenance and with normal ageing of the hardware. However, the first prerequisite to evaluate results is proper knowledge and handling of these limitations, and that is what we tried to do here for standard instrumentation. A similar approach should be taken also with differently designed CD spectrometers operating in the UV-Vis range, such as the pseudo double-beam apparatus by Olis, well described by Sutherland ${ }^{10}$ and the synchrotron radiation $\mathrm{CD}$ beamline-based spectrometers, where the only concerns reported so far have been about $\mathrm{CD}$ signal intensity.

Chirality DOI 10.1002/chir 


\section{ACKNOWLEDGMENTS}

The authors thank Professor Alison Rodger, University of Warwick, UK, for providing us the spectra for the stand$\operatorname{ard~} \mathrm{Na}[\mathrm{Co}$ (EDDS)] (D in Fig. 2).

\section{LITERATURE CITED}

1. Tuzimura K, Konno T, Meguro H, Hatano M, Murakimi T, Kashiwabara K, Saito K, Kondo Y, Suzuki TM. A critical study of the measurement and calibration of circular dichroism. Anal Biochem 1977;81:167-174.

2. Schiffmann DA, Butterfield DM, Yardley RE, Knight A, Windsor SA, Jones C. Val-CD best practice guide A: report CD spectroscopy: an inter-laboratory study. Teddington, UK: NPL DQL-AS009; 2004.

3. Hennessey JH, Curtis Johnson WC. Experimental errors and their effect on analyzing circular dichroism spectra of proteins. Anal Biochem 1982;125:177-188.

4. Kelly SM, Jess TJ, Price NC. How to study proteins by circular dichroism. Biochim Biophys Acta 2005;1751:119-139.

5. Greenfield NJ. Using circular dichroism spectra to estimate protein secondary structure. Nat Protoc 2007;1:2876-2890.

6. Burgess C, Frost T, editors. Standard and best practice in absorption spectrometry. Oxford: Blackwell Science; 1999.

7. Castiglioni E. Spettrofotometria UV/vis criteri di valutazione e controllo. Milano: Morgan Edizioni Tecniche; 1996.

8. Chen FZ, Judge DL, Wu CYR, Caldwellet J. Low and room temperature photoabsorption cross sections of $\mathrm{NH}_{3}$ in the UV region. Planet Space Sci 1998;47:261-266.

9. Knauss HP, Ballard SS. Rotational structure of the Schumann-Runge bands of oxygen in the vacuum region. Phys Rev 1935;48:796-799.

10. Sutherland JC. Measurement of circular dichroism and related spectroscopies with conventional and synchrotron radiation light sources. In: Wallace BA, James RW, editors. Modern technique for circular dichroism and synchrotron radiation circular dichroism spectroscopy. Amsterdam: IOS Press; 2009. p 19-72.

11. Miles AJ, Wallace BA. Calibration techniques for circular dichroism and synchrotron radiation circular dichroism spectroscopy. In: Wallace BA, James RW, editors. Modern technique for circular dichroism and synchrotron radiation circular dichroism spectroscopy. Amsterdam: IOS Press; 2009. p 73-90.

12. Pearse RWB, Gaydon AG. The identification of molecular spectra Wiley: New York; 1950

13. Davidsson A, Norden B. On the problem of obtaining accurate circular dichroism. Calibration of circular dichroism spectrometers. Spectr Acta A 1976;32:717-722.

14. Cassim JY, Yang JT. A computerized calibration of circular dichrometer. Biochemistry 1969;8:1947-1951.

15. Chen CG, Yang JT. Two point calibration of circular dichrometer with d-10-camphorsulfonic acid. Anal Lett 1977;10:1195-1207.

16. Takakuwa T, Konno T, Meguro H. A new standard substance for calibration of circular dichroism: ammonium d-10-camphorsulfonate. Anal Sci 1985;1:215-218

17. European Pharmacopoeia 5.0 Method. 2.2.41. Circular dichroism; EDQM - Council of Europe, 7 allée Kastner: F-67081 Strasbourg, France; 2005. p 63-64.

18. Oakberg TC, Trunk J, Sutherland JC. Calibration of photoelastic modulators in the vacuum UV. SPIE Proc 2000;4133:101-111.

19. Krieger WC, Pschigoda LM. Circular dichrometer calibration by Kramers-Kronig transform methods. Anal Chem 1971;43:675-677.
20. Konno T, Meguro H, Tuzimura K. D-pantolactone as a circular dichroism (CD) calibration. Anal Biochem 1975;67:226-232.

21. McCaffery AJ, Mason SF. The electronic spectra, optical rotary power and absolute configuration of metal complexes the dextro-tris (ethylenediamine) cobalt (III) ion. Mol Phys 1963;6:359-371.

22. DeTar DF. Suggested preliminary standards for calibration of optical rotary dispersion and circular dichroism instruments. Anal Chem 1969;41:1406-1408.

23. Konno T, Meguro H, Murakami T, Hatano M. A critical study on circular dichroism measurement in longer side of visible region. Chem Lett 1981;10:953-956.

24. Steinberg IZ. Device for calibrating instrument that measure circular dichroism or circularly polarized luminescence. US Patent 4,003,663, 1977.

25. Tanaka M, Watanabe KY, Kanedo F, Nakagawa K. Accurate and quick calibration method for polarization-modulation spectroscopy using an ac-modulated polarizing undulator. Rev Sci Instrum 2008;79:083102.

26. Nafie LA, Keiderling TA, Stephens PJ. Vibrational circular dichroism. J Am Chem Soc 1976;98:2715-2723.

27. Schippers PH, Dekkers HPJ. Direct determination of absolute circular dichroism data and calibration of commercial instruments. Anal Chem 1981;53:778-782.

28. Nordén B, Seth S. Critical aspects of measurement of circular and linear dichroism: a device for absolute calibration. Appl Spectr 1985;39: 647-655.

29. Holzwarth G. Circular dichroism measurements to $185 \mathrm{~nm}$ in a commercial recording spectrophotometer. Rev Sci Instrum 1965;36:59-63.

30. Holzwarth G. The Ultraviolet Optical Properties of Polypeptides, PhD Thesis, Harvard University, Cambridge, MS, 1964.

31. Holzwarth G, Doty P. The ultraviolet circular dichroism of polypeptides. J Am Chem Soc 1965;87:218-228.

32. Yamamoto A, Kodama S, Matsunaga A, Hayakawa K, Kitaoka M. Multi-beam circular dichroism detector for HPLC. Analyst 1999;124:483-485

33. Miles AJ, Wien F, Lees JG, Rodger A, Janes RW, Wallace BA. Calibration and standardization of synchrotron radiation circular dichroism and conventional circular dichroism spectrophotometers. Spectroscopy 2003;17:653-661.

34. Miles AJ, Wien F, Lees JG, Wallace BA. Calibration and standardization of synchrotron radiation circular dichroism and conventional circular dichroism spectrometers. II. Factors affecting magnitude and wavelength. Spectroscopy 2005;19:43-51.

35. Inoue Y, Borovkov V, Wada A. Standard sample for test and/or calibration of circular dichroism dispersion meter and UV-visible spectrophotometer. US Patent US2009/0,198,454, 2009

36. Damianoglou A, Crust EJ, Hicks MR, Howson SE, Knight A, Ravi J, Scott P, Rodger A. A new reference material for UV-visible circular dichroism spectroscopy. Chirality 2008;20:1029-1038.

37. Castiglioni E, Lebon F, Longhi G, Gangemi R, Abbate S. An operative approach to correct $\mathrm{CD}$ spectra distortions due to absorption flattening. Chirality 2008;20:1047-1052.

38. Breeze RH, Ke B. A circular dichroism spectrophotometer using an elasto-optic modulator. Anal Biochem 1972;50:281-303.

39. Yang JT, Samejima T. Optical rotary dispersion of catalase. J Biol Chem 1963;238:3262-3267.

40. ASTM E387-84 (1995). Standard test method for estimating stray radiant power ratio of spectrophotometers by the opaque filter method in Annual book of ASTM standards 03.06:740-749, West Conshohocken, PA, 1997. 